\title{
Environmental safety of the gas distribution system
}

\author{
Elena Solovyeva ${ }^{1, *}$ \\ ${ }^{1}$ Moscow State University of Civil Engineering, Yaroslavskoe shosse, 26, Moscow, 129337, Russia
}

\begin{abstract}
This article analyzes the causes of accidents on overhead gas pipelines. Special attention is paid to the operating conditions of gas distribution and gas consumption systems. The main measures aimed at reducing the environmental danger of gas supply systems operation have been identified. The main methods of increasing the reliability of gas distribution systems in the design of gas networks are considered. The complex index of reliability of gas distribution systems is used, which allows choosing the optimal version of the gas supply system.
\end{abstract}

\section{Introduction}

The main task of the gas distribution system is the hourly gas supply to consumers. In addition, it is necessary to exclude the possibility of gas leakage from underground gas pipelines, which may contribute to the formation of gas-air mixture.The reliability of the distribution system of gas supply is the ability to transport the required amount of gas for consumers. The main criterion for the reliability of gas distribution systems is the probability of their failure-free operation during a given time $P(t)$.

Another distinctive feature of gas distribution systems is their social character, as they serve people and ensure their normal livelihoods. The social character is that in case of accidental failures and the cessation of gas supply to consumers, there is not only economic, but also moral damage. When gas supply to buildings is cut off, the air temperature in the rooms decreases, which leads to disruption in the life of people, and the number of diseases increases. In the design of gas distribution systems, the following methods are used: the transition from a dead-end to a ring network, sectioning, duplication, the use of schemes that exclude disconnection of consumers from the gas distribution system. The gas distribution system is characterized by a considerable extent and a high degree of wear. Therefore, it is important to study the causes of accidents on underground gas pipelines. Emergency situations occur in all countries, but during a certain period of time their causes and character change. The development of science, new structures make demands on their calculation, design and installation. If the causes of emergency situations are disclosed correctly and objectively, this significantly reduces the likelihood of their damage. According to the Set of rules 42.101.2003 General provisions for the design and construction of gas distribution systems of metal and polyethylene pipes (2004), in many developed countries, centers for the collection, study and dissemination of information on

\footnotetext{
*Corresponding author solovyva@mail.ru
} 
accidents and issuing recommendations for their prevention have been established and are functioning [1].

An analysis evidences that up to $90 \%$ of accidents take place annually on the operated objects, and in most cases the cause of such malfunctions is a violation of the legislation of town-planning activity during engineering surveys, architectural design and construction and installation works. The system of gas consumption and distribution is one of the most dangerous. All elements of gas distribution and consumption systems require extremely serious attitude, strict observance of norms and rules. If these requirements are not met, explosions of the gas-air mixture occur, accompanied by fires and destruction. The increase in the number of emergency situations associated with the use of natural gas made it necessary to pay serious attention to the state of the elements of gas distribution and consumption systems and to take measures to increase safety [2].

Previously, pipes entered the cellars, from where they got into the apartments by risers. In this case, gas leakage led to its accumulation in the basement, and then to an explosion. Therefore, gas pipes now leave the ground along the outer wall of the house and get it at the level of the first or second floor, which excludes the possibility of gas entering the basements. The most frequent cause of gas leakage and subsequent explosions is a violation of the operation rules. Typical violations are unauthorized redevelopment of apartments and the transfer of gas communications performed unskilled. According to the Decree of the Government of the Russian Federation "On approval of technical regulations on the safety of gas distribution and gas consumption networks" (October 29 2010. No. 870), to prevent the occurrence of gas leaks, it is necessary to pay attention to the reliability of gas distribution and consumption systems. Reliability of gas distribution systems and gas consumption means the ability to transport the required amount of gas to consumers in compliance with specified parameters under normal operating conditions for a certain period of time.

\section{Methods}

There are two main ways to improve reliability when designing gas distribution and consumption systems.

The first way is to improve the quality of the elements that make up the system. Here we mean the use of PE pipes, more durable insulating materials, improving the quality of construction and installation work. The second way provides various ways to improve reliability by installing additional deactivating devices, using duplicate gas pipelines, using ring networks, connecting consumers according to a scheme that excludes the disconnection of consumers from the gas distribution system [3].

\section{Results}

Reliability of systems is estimated by probability-time characteristics. The probabilistic nature of random variables is expressed by the distribution law, which establishes a connection between its possible values and the corresponding probabilities. The laws of distribution of a random variable $\varepsilon_{6}$ are reflected by the distribution function

$$
F_{\varepsilon}(x)=P(\epsilon<x)
$$

The distribution function of a random variable is the probability that $\varepsilon_{0}$ will take a value less than $\mathrm{x}$. The distribution function $F_{\varepsilon}(x)$ is a nondecreasing function of the argument $\mathrm{x}$, therefore, for $x_{2}>x_{1}, F_{\varepsilon}\left(x_{2}\right) \geq F_{\varepsilon}\left(x_{1}\right)$. At minus infinity, the function becomes equal to zero, and at plus infinity it is equal to $F_{\varepsilon}(+\infty)=1$. 
An example of the distribution functions of a continuous random variable is the time of the element repair to failure. Average time of work, characterizing the average level of the element quality, is determined by the mathematical expectation of the element's operating time to failure. But due to the random nature, the lifetime of each element will differ to some extent from the mean. This difference reflects the distribution law.

In the initial period of operation, the probability of failure is small and the value of $F_{\tau}(t)$ grows slowly. In the middle part, the curve rises steeply. The greater the slope of the curve, the smaller difference between performance characteristics of the elements. In the final part, the curve is also quite canopy, as the probability of a prolonged operation of the element is much larger than the average value, which is very small. The probability that the lifetime of an element is less than $t$ is determined by the function e $F_{\tau}(t)$, hence, e $F_{\tau}(t)$ characterizes the reliability of the element, since e $F_{\tau}(t)$ is the probability of failure of the element, up to the moment e $F_{\tau}(t)=P(\tau<t)$..

The probability of failure of the element greater than the time $t$ is determined by the expression

$$
P_{\tau}(t)=1-F_{\tau}(t)=P(\tau>t)
$$

This probability is called the reliability function. It is the main criterion of reliability, characterizing the failure-free operation of the element for a specified period of time.

For gas distribution systems, the failure flow parameter " $\omega$ " is an important parameter. It is used to determine the statistical data of damages recorded by operating organizations. If during the whole observation period, $\Delta \mathrm{t}$ is usually taken to be 1 year, each element of $\mathrm{N}$ observed failed mi times, then

$$
\omega=\sum_{i=1}^{N} m_{i} /(N \Delta t)
$$

The inverse of the failure flow parameter $T=1 / \omega$, measured in years, is the time to failure. The parameter of the pipeline failure flow is referred to $1 \mathrm{~km}$ length. In the case $\omega=\omega_{\Gamma} l$, where $\omega_{\Gamma}$ is the parameter of the flow of failures, referred to $1 \mathrm{~km}$ and measured in $1 /$ year $\mathrm{km} ; l$ is the length of the gas pipeline, $\mathrm{km}$.

Analysis of the data of the failure flow parameters allowed determining the following values of the parameter of sudden failures, which are the basis for calculating the reliability of systems, $1 / \mathrm{km}$ year

1) Corrosion damage

$\omega_{\mathrm{p}}=1,5410^{-3} 1 /(\mathrm{km}$ year $)$;

2) Damage to welded joints

$\omega_{\mathrm{p}}=0,9210^{-3} \quad 1 /(\mathrm{km}$ year $)$;

3) Mechanical damage

$\omega_{\mathrm{p}}=2,0910^{-3} 1 /(\mathrm{km}$ year $)$;

The mathematical expectation of the characteristic of functioning quality is determined by the formula

$$
Q(t)=M Q_{x}(t)=Q_{0} l^{-\sum \omega_{i} t}+\sum_{i=l}^{i=1} Q_{i} \frac{\omega_{i}}{\sum \omega_{i}}\left(1-e^{-\sum \omega_{i} t}\right)
$$

Using relation $Q_{i}=Q_{0}-\Delta Q_{j}$ transform the resulting formula

$$
Q(t)=Q_{0}-\sum_{i=l}^{i=1} \Delta Q_{i} \frac{\omega_{i}}{\sum \omega_{i}}\left(1-e^{-\sum \omega_{i} t}\right)
$$

The formula for the reliability index of the system will be

$$
R_{\text {sist }}=1-\sum_{i=l}^{i=1} \frac{\Delta Q_{j} \omega_{i}}{Q_{0} \sum \omega_{i}} \cdot\left(1-e^{-\sum \omega_{i} t}\right)
$$


where $M Q_{x}(t)$ is a mathematical expectation of disconnected power in emergency situations; $\sum \omega_{i}$ is a sum of failure flow parameters of all elements of the ring network; $\Delta Q_{j}$ is a disconnected power; $Q_{0}$ is an estimated gas consumption.

To increase the reliability and safety, steel pipes are replaced with polyethylene ones, and this solves the problem of corrosion. Polyethylene pipes are used in the reconstruction of worn-out steel pipes without opening them (hauling method).

Technical regulations "On the safety of buildings and structures" does not provide for the use of technical security equipment. This document provides for stricter requirements only for the operation of house gas equipment. The most reliable way to increase safety is to equip the gas-consuming equipment with a flame-extinguishing control system (gascontrol). Other security systems (sensors - gas analyzers, solenoid valves, thermo-shut-off valves) require much higher costs.

In Europe, the use of shut-off valves for the consumption of gas-stop gas is widely used. The use of valves will prevent gas leakage due to gas pipeline rupture or partial or complete destruction of the valve. In the developed SRT "Safe connection of residential buildings to gas networks" only valves for external gas pipelines are taken into account, although there are valves for consumption for internal gas pipelines.

According to the Decree of the Government of the Russian Federation "On measures to ensure safety when using and maintaining indoor and in-apartment gas equipment" (May 14 2013. No. 410), valves on external gas pipelines-inputs protect buildings from possible fires and explosions as a result of sharp depressurization of a gas pipeline or valves near the building or its facade [4-6].

In Europe, in addition to the traditional regulators on the external gas pipelines, individual pressure regulators (reducers) are used on internal gas pipelines immediately before the gas-using equipment. These regulators-reducers are adjusted to the specific working pressure of gas-using equipment, ensuring its optimal operation with the maximum efficiency and minimum content of harmful substances in the combustion products. Cabinet control points are used is not only in wall-mounted or stand-alone versions, but also on the underground gas pipelines. They can be also built into the wall of the building.

\section{Discussion}

In addition to the reliability indicators, it is necessary to consider the reserve for reducing the cost of gas distribution networks - to replace low-pressure networks with networks of medium pressure.

In gas pipelines, the pressure loss is inversely proportional to the average gas pressure. Therefore, with an increase in pressure, the diameters of gas pipelines will decrease as well as the cost of the network. The diameter of the pipeline is determined from the expression:

$$
\begin{gathered}
P_{n}^{2}-P_{k}^{2}=a \frac{Q^{2}}{d^{5.25}} \\
d=\left(\frac{a}{2}\right)^{0.19} \frac{Q^{0.38}}{\Delta P^{0.19} P_{s r}^{0.19}}
\end{gathered}
$$

where $P_{n}, P_{k}$ is initial and final high pressure in the pipeline;

$Q$ is gas consumption;

$d$ - gas pipeline diameter;

$a$ - coefficient depending on the properties of the gas, the material of the pipeline and its length;

$\Delta P$ - gas pressure fall in the network; 


$$
P_{n}^{2}-P_{n}^{2}=a \frac{Q^{2}}{d^{5.25}}
$$

When gas pressure increases, taking capital investments in gas pipelines proportional to their diameters, for sections of the network with the same gas consumption, the decrease in capital investment is determined by the dependence obtained from the following formula:

$$
K_{v . S}=K_{n}\left(\frac{P_{n}}{P_{v . s}}\right)^{0,19}\left(\frac{\Delta P_{n}}{\Delta P_{v . s}}\right)^{0,19}
$$

where $K_{v . s}, K_{n}$ - capital investment in high and low pressure networks;

$P_{n}$ and $P_{v . s}-$ low and high gas pressure respectively;

$\Delta P_{v . s}$ and $\Delta P_{n}-$ pressure fall.

With increasing gas pressure, the calculated differential fall also increases, which leads to a decrease in the cost of the network. [7,8].

The main reserve for reducing the cost of urban gas networks is to replace low-pressure networks with networks of medium pressure.

According to the degree of replacement there are three options:

1. A system with gas control points, in which the main gas streams are transported over networks of medium pressure, for small consumers, gas is transported over low pressure gas pipelines.

2. Systems with quarterly regulatory points, in which gas pipelines of medium and high pressure constitute the main part of quarterly regulatory points with low productivity.

3. Systems with district regulatory points. They include external gas pipelines of high and medium pressure. Regulators are installed separately for each house in the wall cabinets. $[9,10]$.

\section{Conclusions}

To prevent fire risk due to small gas leaks inside the building, a number of measures are envisaged:

- Installation of gaseous analyzers, which determine the concentration of combustible gases in the premises and signal to the control center and to the executive locking body in case of permissible concentrations exceeding.

- Installation of fire detectors detecting an increase in temperature and smoke in the room and giving a signal to the control center and to the executive locking body in case of permissible concentrations exceeding.

- Installation of a solenoid valve at the entry of a gas pipeline into the building, which cuts off the gas supply when a signal from the gassing sensor or fire alarm is received, or from the control room.

- Installation of the thermo-shut-off valve on the supply pipeline to the gas-using equipment when the temperature is exceeded.

Passive measures to increase security include limiting access by unauthorized persons to the internal gas pipeline, connections and fittings:

- Laying a gas pipeline in a ventilated reservoir or mine, with the possibility of access to the pipeline by the personnel of the operating organization;

- fixed joint of the gas pipeline;

- Restriction of access to the plug connection and fittings.

The safety of gas supply to residential buildings in Russia is at a low level due to the deterioration of steel gas distribution systems. The updated version of SNiP 42-01-2002", where a special attention is paid to safety issues, should contribute to the adoption of measures to improve the level of safety in gas supply systems $[9,10]$. 


\section{References}

1. W. Lin, A. Gu, Tianranqi Gongye 5, 87-91 (2001)

2. T. Gjesdal, Computing and Visualization in Science 1-2, 25-32 (2000)

3. J.R. Puskar, Engineered Systems 9, 54-59 (2004)

4. V. Safonov, S. Kovalev, S. Ovcharov, A. Melnikov, Proceedings of the European Safety and Reliability Conference 2006, ESREL 2006 - Safety and Reliability for Managing Risk European Safety and Reliability Conference, 2519-2524 (2006)

5. J. Kudo, M. Takahashi, T. Wakabayashi, N. Sakaba, Transactions of the Atomic Energy Society of Japan 4, 360-367 (2010)

6. S.N. Nikolaenko, A.S. Dyundik, Journal of Economics and Social Sciences 9, 13-15 (2017)

7. N. Meshkati, Automation and Human Performance: Theory and Applications 1, 427446 (2018)

8. Ye.B. Solovyeva, International Journal of Mechanical Engineering \& Technology (IJMET) 9(2), 761-76 (2018)

9. V. Zhila, Ye.B. Solovyeva, E3S Web of Conferences 33, 02066 (2018)

10. V. Zhila, E. Solovyeva, International Science Conference SPbWOSCE-2017 "Business Technologies for Sustainable Urban Development, 3-16 (2018) 